\title{
Neuroprotective Effect of Combined Therapy of \\ Liraglutide with Vitamin D on Streptozotocin- Induced Diabetic Neuropathy in Rats.
}

\author{
Mohamed Mostafa Elbadr ${ }^{1}$, Mona Sedeek ${ }^{2}$, Mohamed A.M. \\ Mostafa $^{3}$-Hala Mostafa El Badre ${ }^{4}$ \\ ${ }^{1}$ Department of Medical Pharmacology, Faculty of Medicine, Assiut \\ University, Assiut, Egypt. \\ ${ }^{2}$ Department of Physiology, Faculty of Medicine, Aswan University, \\ Aswan, Egypt. \\ ${ }^{3}$ Anaesthesia, ICU and Pain Relief Department, South Egypt Cancer \\ Institute, Assuit University, Egypt. \\ ${ }^{4}$ Department of Medical Biochemistry, Faculty of Medicine, Assiut \\ University, Assiut, Egypt.
}

\begin{abstract}
Background:

Liraglutide is an incretin mimetic agent that approved recently in type- 2 diabetes. The use of vitamin D was reported to be associated with an improvement in diabetic neuropathy.

The aim of the study:

Evaluation of the effect of liraglutide and vitamin D each alone and in combination with each other on diabetic neuropathy induced by streptozotocin in rats.

Materials and Methods:

Five groups of Wistar rats were used in the experiment. Diabetic neuropathy was induced in groups 2, 3, 4, and 5 using a single intraperitoneal(i.p.) injection of streptozotocin(STZ) in a dose of $60 \mathrm{mg} / \mathrm{kg}$. The third, fourth, and fifth groups were treated for 4 weeks with liraglutide $(0.8 \mathrm{mg} / \mathrm{kg})$, vitamin $\mathrm{D}(12 \mu \mathrm{g} / \mathrm{kg})$, and combination of the 2 drugs, respectively. Then the behavioral tests were done (hot plate, tail-flick, paw withdrawal pressure, and the rotarod tests). Blood samples were used for assessment of blood glucose, tumor necrosis factor $-\alpha$ (TNF- $\alpha$ ) and interleukin-I $\beta(\mathrm{IL}-\mathrm{I} \beta)$.Malondialdehyde(MDA) and glutathione (GSH)were measured in the sciatic nerve homogenate.
\end{abstract}

\section{Results:}

Liraglutide caused significant improvement in the behavioral tests of the diabetic rats with a significant reduction in blood glucose, TNF- $\alpha$, IL-1 $\beta$, and malondialdehyde. Vitamin D caused mild improvement in the behavioral tests, inflammatory and oxidative stress markers. The combined use of liraglutide with vitamin D caused more improvement in diabetic neuropathy tests, inflammatory and oxidative stress markers.

\section{Conclusion:}

Liraglutide has a moderate neuroprotective, anti-inflammatory, and antioxidant effects in cases of streptozotocin-induced diabetic neuropathy which are enhanced by the addition of vitamin $\mathrm{D}$.

Keywords: Diabetic neuropathy;Liraglutide; Streptozotocin; Vitamin D 


\section{INTRODUCTION}

Diabetic neuropathy is one of the microvascular complications that occur with type-I and type-II diabetes mellitus after a long time of the disease. The neuropathy starts with loss of the sensation in the limbs and followed by ulceration of the foot that may end in amputation of the affected limb [1]. After 10-20 years of the development of diabetes, more than $50 \%$ of the patients may develop neuropathy. Neuropathy is initiated by long term uncontrolled hyperglycemia, that stimulates several reactions such as oxidative phosphorylation, aldose reductase, and non-enzymatic glycation with alteration in protein kinases [2]. These reactions cause stimulation of oxidative stress with excess production of reactive oxygen species and toxic metabolites [3]. The excessive formation of these products causes damaging the DNA of the mitochondrial dorsal root ganglion that causes microangiopathy with nerve ischemia and malfunction of the peripheral nerves [4]. Glycolysis causes stimulation of protein kinase$\mathrm{b}(\mathrm{PK}-\mathrm{b})$ and $\mathrm{c}$ (PK-c) causing upregulation of gene expression of several cytokines. Reactive oxygen species together with the end products of glycolysis stimulate nuclear factorkappa B. This leads to an increase in the transcription of several inflammatory mediators such as chemokines, cytokines, adhesion molecules, cyclooxygenase- 2 and inducible nitric oxide synthase. The excess production of these inflammatory mediators is responsible for peripheral neuropathy development [5].

Liraglutide is anincretin mimetic agent that was approved as an adjuvant therapy to exercise and diet in diabetes mellitus type-2 [6]. The drug is a longacting human glucagon-like peptide type-1 (GLP-1) analog. It has a 97\% homology with endogenous human GLP-1 which is obtained by replacing arginine by lysine amino acid at position 34. Endogenous GLP-1 is metabolized rapidly by the enzyme dipeptidyl peptidase-4 (DPP-4), so it has a short duration of action. However,liraglutide has a prolonged half-life as it has a fatty acid part that attaches to the albumin [7]. GLP-1 receptors are found mainly in the alpha and beta cells of the pancreas, the heart, the lung, the GIT, and the nervous system. In cases of hyperglycemia, liraglutidestimulates insulin secretion by increasing cAMP and inhibits the secretion of glucagon [8]. Liraglutide delays the emptying of the stomach, reduces the appetite and causes a reduction of the body weight, and approved by the FDA as a weightreducing agent. It also reduces the obesity-induced metabolic disorders and cardiovascular risk factors and improves the lipid profile. The drug has also an antioxidant and antiinflammatory effects [9].

Vitamin D has an important role in the pathogenesis of several autoimmune diseases and the prevention of breast, prostatic and colorectal cancers as it can prevent the proliferation of the cancer cells. Although vitamin D has an important role in the homeostasis of calcium, many reports indicated that it has a role in both types of diabetes mellitus [10]. There is a strongassociation between the deficiency of vitamin $\mathrm{D}$ and the changes in the level of insulin, blood glucose, and the insulin sensitivity of the tissues [11]. Vitamin D may have a role in the control of diabetes mellitus by enhancing tissue sensitivity to insulin and pancreatic insulin release. Vitamin D may affect multiple functions in the body and its receptor is distributed in more than thirty tissues including the pancreas, the 
lymphocytes, and theheart [12]. Many reports have shown a negative association between glucose intolerance and the serum levels of vitamin $\mathrm{D}$; however other studies showed no association between both conditions.Several animal studies indicated that there is a positive effect on the addition of vitamin $\mathrm{D}$ on the control ofhyperglycemia in diabetic animals [13]. Vitamin D receptors and 1- $\alpha$ hydroxylase enzyme which responsible for vitamin $\mathrm{D}$ activation are expressed on the beta cells of the pancreas suggesting that vitamin $\mathrm{D}$ may have a role in glucose homeostasis. Vitamin D could be used as an adjunct treatment in the management of diabetes mellitus if there is an approval for its beneficial effect in the control of hyperglycemia [14]. A recent study demonstrated that there is a reduction in the level of vitamin $\mathrm{D}$ in patients with painful diabetic peripheral neuropathy [15]. Vitamin D (50,000 IU) orally once / week for 3 months is associated with an improvement of the signs and symptoms of diabetic neuropathy. Vitamin D level in the serum should be monitored for the patients with diabetic neuropathy and a low level of the vitamin should be treated to reduce the severity of the neuropathy [16]. The aim of the study was the evaluation of the effect of liraglutide and vitamin $\mathrm{D}$ each alone and in combination with each other on the diabetic neuropathy induced by streptozotocin and their effects on the inflammatory and oxidative stress markers in rats.

\section{MATERIALS AND METHODS}

\subsection{Drugs and chemicals}

\section{Liraglutidewas} obtained

NovoNordisk $\mathrm{mg} / \mathrm{ml})$. Vitamin

\section{D3}

(Victoza, 6

(Cholecalciferol) was purchased from Sigma-Aldrich, USA, and dissolved in corn oil. Streptozotocin was purchased from Sigma-Aldrich, USA. RatTNF$\alpha$ ELISAKit (cat numberab100784, lot number GR307317-1, AbcamBiochemicals, Cambridge, UK)) and rat IL-I $\beta$ ELSIA kit (Ray Biotech, USA) were used for determination of the level of TNF- $\alpha$ andIL-I $\beta$, respectively in the sciatic nerve homogenate. Glucose oxidase commercial kit (Sigma, St. Louis, Mo, USA) was used for measuring the serum glucose. Ellman's reagent, reduced glutathione, and malondialdehyde were obtained from Sigma Aldrich, USA. 2-thiobarbituric acid and phosphate-buffered saline were purchased from MP Biomedical, France. The rest of the chemicals were of analytical grade and obtained from the local commercial sources.

\subsection{Animals}

Thirtymale Wistarrats with a weight of 150 - $200 \mathrm{~g}$ were usedin the research. The animals were purchased from the animal houseof the Faculty of Medicine,Assiut University. The rats consumed the ordinary laboratory food and water ad libitum. For adaptation, the rats were kept in the laboratory for seven days beforethe start of the experiment. The experiment was conducted accordingto the guide for the care and use of laboratory animals of the National Institutes of Health (NIH 1985). The research was approved by the ethics committee of the College of Medicine, Assiut University

\subsection{Experimental design}

\subsubsection{Induction of diabetes and diabetic neuropathy}

Diabetes was induced in rats of groups $2,3,4$, and 5 by single intraperitoneal (i.p.) injection of streptozotocin (STZ) in a dose of $60 \mathrm{mg} / \mathrm{kg}$ b.w. [17]. STZ was dissolved in $10 \mathrm{mM}$ of ice-cold sodium citrate buffer with $\mathrm{pH}$ 4.5. The solution was kept in ice and used 
within5 minutes of its preparation [18]. Animals were fasted overnight before giving the STZ. Blood samples were collected from the rat tail vein after 72 hours of STZ injection and measuring of blood glucose was done. Rats with blood glucose 200-300mg/dl were considered diabetic and were taken in the experiment [18]. Diabetic rats were testedfor hyperalgesia using the hot plate test and tail-flick test after 4 weeks of induction of diabetes. Diabetic rats that developed significant hyperalgesia were considered as rats with diabetic neuropathy and included in the study [17].

\subsubsection{Animal grouping}

Five groupsof animals, 6rats in each group were used in the experiment. The first group wasinjected i.p. with $1 \mathrm{ml}$ of $10 \mathrm{mM}$ of ice-cold sodium citrate buffer with $\mathrm{pH}$ 4.5. and used as a control non-diabetic group. The second group was injected by a single i.p. injection of streptozotocin (STZ) in a dose of 60 $\mathrm{mg} / \mathrm{kg} \mathrm{b.w}$. and used as a control diabetic group. The third group was diabetic rats which developed diabetic neuropathyand started treatment after 4 weeks of STZ injection with liraglutide $0.8 \mathrm{mg} / \mathrm{kg}$ s.c daily for 4 weeks. The fourth group of rats had diabetic neuropathyand treated with vitamin D12 $\mu \mathrm{g} / \mathrm{kg} /$ day orally for 4 weeks using an oral gavage needle. The fifth group of rats had diabetic neuropathy and treated with a combination ofliraglutide $(0.8 \mathrm{mg} / \mathrm{kg}$ s.c daily) and vitamin D3 (12 $\mu \mathrm{g} / \mathrm{kg} /$ day orally) for 4 weeks.

The selection of the liraglutide and vitamin $\mathrm{D}$ doses depended on the range of doses of the previous investigations $[19,20]$. After the end of 4 weeksof treatment, the behavioral tests were done (hot plate, tail-flick, paw pressure, and the rotarod tests). Then the animals were anesthetized using pentobarbital $50 \mathrm{mg} / \mathrm{kg}$ b.w.followed bydecapitation of rats by cervical dislocation. Blood samples were collected and centrifuged for 10 minutes at 3000 revolutions/minuteand storedat

$-20{ }^{\circ} \mathrm{Ctill}$ the use forthe assessment of the blood glucose and the proinflammatory markers (TNF- $\alpha$ and IL-I $\beta$ ). The sciatic nerves of both sideswere removed and weighed then frozen in liquid nitrogen till the use for the measuring of markers of oxidative stress. Later on sciatic nerves, homogenization in phosphate buffer was done. Then cold centrifugation for 15 minutes at 10500 revolutions/minute was performed. Then MDA andreduced glutathione were measured in the sciatic nerve homogenate.

\subsection{Evaluation of the behavioral activity}

2.4.1. Thermal stimulus: hot-plate test

The hot plate test was used for the evaluation of the effect of the different treatments on the thermal pain threshold according to the method that was prescribed by Hunskaar et al[21]. The rat was placed on the hot-plate at a constant temperature of $55{ }^{\circ} \mathrm{C}$. The reaction time was measured. It was the time from the application of heat stimulation to the response caused by the animal in the form of licking of the hind paws or jumping. The cut off time was 20 seconds to prevent any injury to the rat tissue. The thermal pain threshold was determined at the start of the experiment, after 4 and 8 weeks of the start of the experiment [21].

\subsubsection{Tail flick test}

The rate of nociception was measured using the tail-flick test as described by Sugimoto and his coworkers (2008). Thermal light was directed to the distal end of the tail of 
the rat using a special apparatus (Model DS 20 Apelex, France). The tail-flick latency which is the time from the application of the light to the sudden withdrawal of the tail of rat was measured. The test was repeated 3 times with 5 minutes interval and the mean of these readings was used for statistical analysis. Cut off time of 15 seconds was used to prevent any tissue injury. The tail-flick test was done at the start of the experiment, after 4 and 8 weeks of the start of the experiment [22].

\subsubsection{Mechanical Hyperalgesic Test (Randall-Selitto paw pressure test)}

The Randall-Selitto electronic analgesia-meter(Ugo Basile, Italy) was used for the assessment of the mechanical hyperalgesia. Before the test, the rat was carefully immobilized and covered by a soft cotton cloth. Then the application of a mechanical force in an increasing manner was done. The tip of the device was inserted into the medial part of the plantar surface of the hind paw. The monitored reaction included paw withdrawal, animal vocalization, or trial of the rat to escape. The measured parameter was the weight for the appearance of thesebehavioral reactions (weight in grams). To avoid any skin damage, the maximum applied force was not more than $250 \mathrm{~g}$. The test was done after 4 weeks of treatment with the tested drugs [23].

\subsubsection{Assessment of motor coordination (Rotarod Test)}

Motor coordination of the rats was measured using the Rotarod apparatus (Ugo Basile, Varese, Italy). The rats were trained for three days before the start of treatment. The time (in seconds) that the trained rats could stay on the rod at a speed of 20 revolutions/ minute was recorded in all rats. Rats that stayed for 180 seconds (cutoff time) on the rotarod bar were included in the experiment. Rats were placed on the rotating rod twice with 5 minutes' interval and the mean of the 2 trials was used for statistical analysis. The rotarod test was done after 4 weeks of treatment with the tested drugs [24].

\subsection{Determination of the blood glucose level}

The determination of blood glucose was done spectrophotometrically by using the glucose oxidase/peroxidase method [25]. The measurement of blood glucose was done after 72 hours of STZ injection. Any animal with blood glucose $200-300 \mathrm{mg} / \mathrm{dl}$ were considered as diabetic one and included in the study. Blood glucose was also measured after 4 weeks of treatment with different drugs.

\subsection{Evaluation of the proinflammatory cytokines}

2.6.1. Determination of the level of tumor necrosis factor-(TNF- $\alpha$ )

The level of TNF- $\alpha$ in rat serum was measured using the rat TNF- $\alpha$ ELISA kit according to the instructions of the manufacturer. The samples were added to the well that coated to TNF- $\alpha$ and gentle shaking was done followed by incubation at $37^{\circ} \mathrm{Cfor} 2 \frac{1}{2}$ hours. Then washing of the plate was done followed by adding a specific antibody for each well with re-incubation for one hour. Washing of the plate was performed, and then we added a streptavidin solution with re-incubation for 45 minutes. After washing of the plate, a one-step substrate reagent was added. In the end, a stop solution was added and the reaction was measured using an automated ELISA reader at $450 \mathrm{~nm}$ [26].

\subsubsection{Determination of the level of

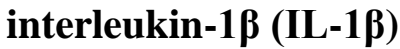

The level of IL-1 $\beta$ in the rat serum was measured using the rat IL$1 \beta$ ELSIA kit according to the instructions of the manufacturer. 
Firstly, we added the samples to microplate wells coated with IL-1 $\beta$ and incubated for 2 hours at $37{ }^{\circ} \mathrm{C}$. Biotinantibody was added after complete removal of the liquid then reincubation for another 1 hour was done. Washing of the plate with wash buffer, then adding of HRP-avidin wasdone with re-incubation for another 1 hour at $37{ }^{\circ} \mathrm{C}$. Washing was done again and

3,3',5,5'-Tetramethylbenzidine (TMB) substrate was added and re-incubated for 15 minutesat $37{ }^{\circ} \mathrm{C}$ in dark. We added a stop solution to the wells and the absorbance was read by ELISA reader at $450 \mathrm{~nm}$ within 5 minutes [26].

\subsection{Evaluation of the oxidative stress markers}

2.7.1. Determination of the malondialdehyde (MDA)

The level of MDA was measured in the rat sciatic nerve homogenate by the method indicated by Ohkawa et al. (1972). MDA level was measured spectrophotometrically after a colorimetric reaction with thiobarbituric acid. MDA was determined because it is a good marker for oxidative stress and peroxidation of lipids [27].

\subsubsection{Determination of the reduced glutathione (GSH)}

The level of GSH in the rat sciatic nerve homogenate was measured according to the method described by BoyneandEllman (1972). Trichloroacetic acid $10 \%$ was mixed with the rat sciatic nerve homogenate then centrifuged at 5000 revolutions/minute at $-4{ }^{\circ} \mathrm{c}$ for 10 minutes. Then disodium hydrogen phosphate buffer ( $\mathrm{pH}$ 8.4) and Ellman's reagent $(0.25 \mathrm{ml})$ were added to the supernatant. The incubation of the samples for 10 minutes was done then the absorbance of the color was measured at 412 nm spectrophotometrically [28].

\subsection{Statistical analysis}

Data were represented as the mean \pm SE of 6 observations. One-way analysis of variance (ANOVA) was done to detect any statistically significant difference between the different groups. For multiple comparisons between the groups, Tukey's post hoc test was used. Twoway analysis of variance (ANOVA) followed by Tukey's post hoc test was used to analyzethe effect of the tested drugs on hot plate test and tail-flick test. If $\mathrm{P}<0.05$, the results were considered as statistically significantdifferences. The analysis wasdoneby the use ofPrism software (Graph-Pad Software, version 7).

\section{RESULTS}

3.1. Results of the evaluation of the behavioral activity

3.1.1. Rat hot plate latency, at the start of the experiment ( 0 Time), after 4 weeks of single i.p. injection of streptozotocin (STZ) $60 \mathrm{mg} / \mathrm{kg}$ (4 Weeks) and after 4 weeks of the daily use of liraglutide (LIRA) 0.8 $\mathrm{mg} / \mathrm{kg}$, vitamin D $12 \mu \mathrm{g} / \mathrm{kg}$ and their combination (8 Weeks).

The results demonstrated that after 4 weeks of single i.p. injection of streptozotocin60 $\mathrm{mg} / \mathrm{kg}$, there was a significant $(p<0.05)$ reduction of the rat hot plate latency in comparison with the control non-diabetic group (Fig. 1). Treatment with liraglutide, vitamin $\mathrm{D}$, or their combination for 4 weekscauseda significant $(p<0.05)$ increase in the hot plate latency of diabetic rats in comparison with the streptozotocin treated group. The increase was more marked with the use ofliraglutide or the use of liraglutide with vitamin $\mathrm{D}$ as there was no significant difference in the hot plate latency between the liraglutide treated groups and the control non-diabetic group(Fig. 1). 


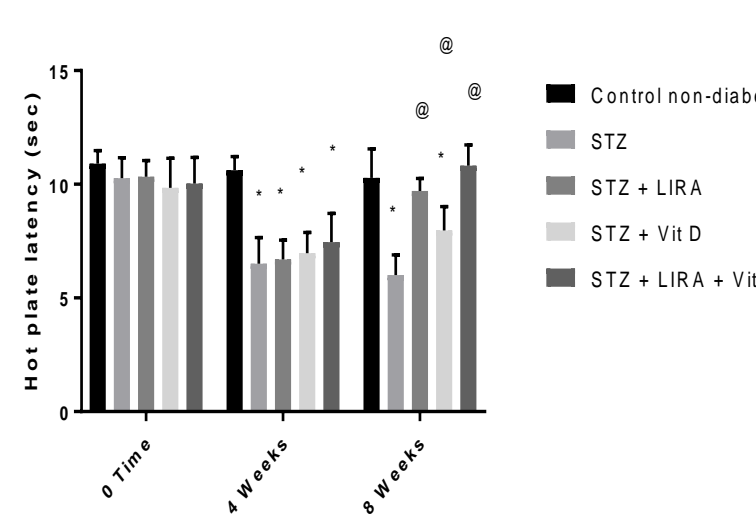

3.1.2. Rat tail-flick latency, at the start of the experiment ( 0 Time), after 4 weeks of single i.p. injection of streptozotocin (STZ) $60 \mathrm{mg} / \mathrm{kg}$ (4 Weeks) and after 4 weeks of the daily use of liraglutide (LIRA) 0.8 $\mathrm{mg} / \mathrm{kg}$, vitamin D (Vit D) $12 \mu \mathrm{g} / \mathrm{kg}$ and their combination (8 Weeks).

After 4 weeks of a single injection of streptozotocin60 $\mathrm{mg} / \mathrm{kg}$, there was a significant $(\mathrm{p}<0.05)$ reduction of the rat tail-flick latency compared to the control non-diabetic group (Fig. 2).

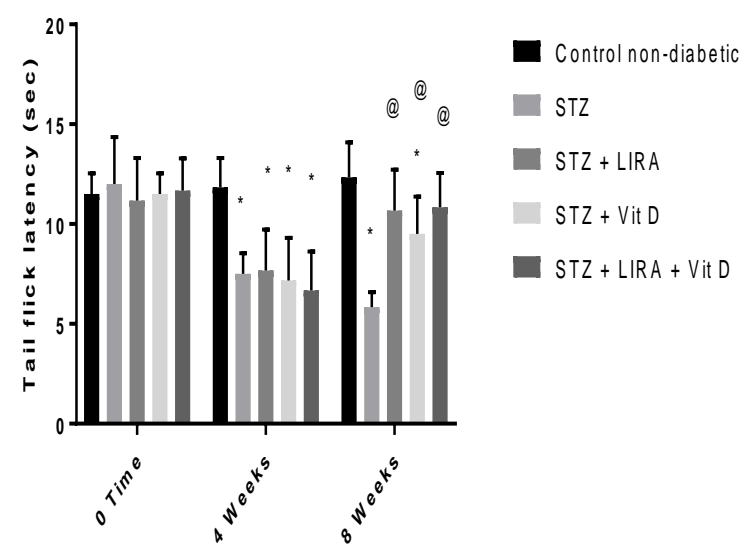

3.1.3. Effect of single i.p. injection of streptozotocin (STZ) $60 \mathrm{mg} / \mathrm{kg}$, liraglutide (LIRA) $0.8 \mathrm{mg} / \mathrm{kg} / \mathrm{day}$ s.c.for 4 weeks, vitamin D (Vit D) 12 $\mu \mathrm{g} / \mathrm{kg} / \mathrm{days}$ orally for 4 weeks and their combination on the rat paw withdrawal pressure.

The results of the study demonstrated that after 8 weeks of single i.p.
Fig.1 Rat hot plate latency, at the start of the experiment ( 0 Time), after 4 weeks of single i.p. injection of streptozotocin (STZ) 60 $\mathrm{mg} / \mathrm{kg}$ (4 Weeks) and after 4 weeks of the daily use ofliraglutide (LIRA) $0.8 \mathrm{mg} / \mathrm{kg}$, vitamin D (Vit D) $12 \mu \mathrm{g} / \mathrm{kg}$ and their combination (8 Weeks).

Results were represented as mean \pm SE (every group consisted of 6 rats)

$* p<0.05$ in comparison with control nondiabetic rats.

${ }^{\circledR} \mathrm{p}<0.05$ in comparison with the STZ treated group.

Four weeks of treatment with liraglutide, vitamin $\mathrm{D}$, or their combination resulted in a significant $(p<0.05)$ increase in the tail-flick latency of diabetic rats in comparison with the streptozotocin treated group. The increase in the tail-flick latency was more marked with the use ofliraglutide than vitamin $\mathrm{D}$ as there was no significant difference in the latency between the liraglutide treated groups and the control non-diabetic group (Fig. 2).

Fig.2 Rat tail-flick latency, at the start of the experiment ( 0 Time), after 4 weeks of single i.p. injection of streptozotocin (STZ) 60 $\mathrm{mg} / \mathrm{kg}(4 \mathrm{Weeks})$ and after 4 weeks of the daily use of liraglutide (LIRA) $0.8 \mathrm{mg} / \mathrm{kg}$, vitamin D (Vit D) $12 \mu \mathrm{g} / \mathrm{kg}$ and their combination (8 Weeks).

Results were represented as mean \pm SE (every group consisted of 6 rats)

$* \mathrm{p}<0.05$ in comparison with the control nondiabetic group.

${ }_{\mathrm{p}}^{\mathrm{p}}<0.05$ in comparison with the STZ treated group.

injection of streptozotocin $60 \mathrm{mg} / \mathrm{kg}$, there was a significant $(\mathrm{p}<0.05)$ reduction of the rat paw withdrawal pressure compared to the control nondiabetic group (Fig. 3). Treatment with liraglutidefor 4 weekscaused a significant $(p<0.05)$ increase in the paw withdrawal pressure of the diabetic rats in comparison with the 
streptozotocin treated group (Fig. 3). Treatment with vitamin $\mathrm{D}$ for 4 weeks caused a non-significant increase in the paw withdrawal pressure of diabetic rats in comparison with the streptozotocin treated group (Fig. 3). Four weeks of treatment with the combination of liraglutide and vitamin $D$ caused a significant $(\mathrm{p}<0.05)$

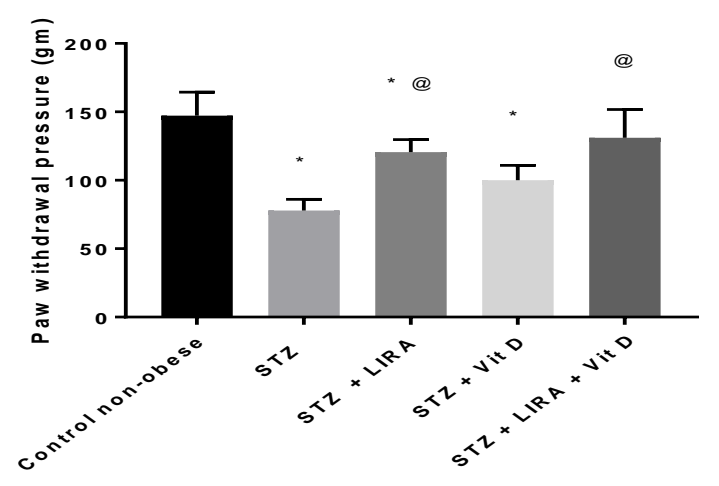

3.1.4.Effect of single i.p. injection of streptozotocin (STZ) $60 \mathrm{mg} / \mathrm{kg}$, liraglutide (LIRA) $0.8 \mathrm{mg} / \mathrm{kg} / \mathrm{day}$ s.c.for 4 weeks, vitamin D (Vit D) 12 $\mu \mathrm{g} / \mathrm{kg} / \mathrm{day}$ orally for 4 weeks and their combination on the rotarod staying time of the rats.

Single i.p. injection of streptozotocin60 $\mathrm{mg} / \mathrm{kg}$ caused a significant $(\mathrm{p}<0.05)$ reduction of the staying time of rats on the rotarod test compared to the control non-diabetic group (Fig. 4). Treatment with liraglutidefor 4 weeks caused a

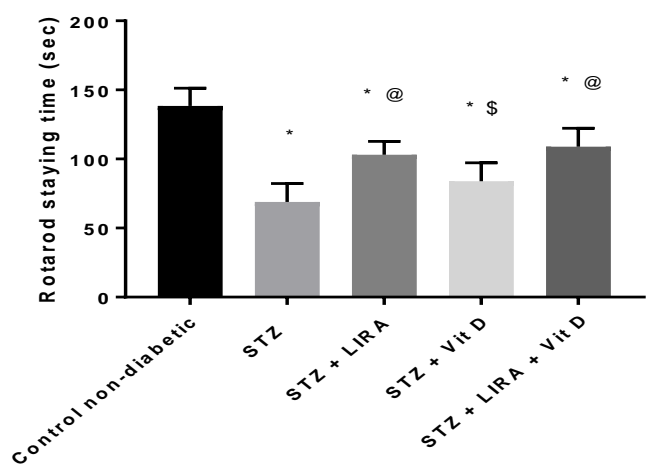

Fig. 4 Effect of single i.p. injection of increase in the paw withdrawal pressure of diabetic rats compared to the streptozotocin treated group and there was no significant difference in the paw withdrawal pressure between the effect of this combination and the control non-diabetic group (Fig. 3).

Fig.3 Effect of single i.p. injection of streptozotocin (STZ) $60 \mathrm{mg} / \mathrm{kg}$, liraglutide (LIRA) $0.8 \mathrm{mg} / \mathrm{kg} /$ day s.c.for 4 weeks, vitamin D (Vit D) $12 \mu \mathrm{g} / \mathrm{kg} /$ days orally for 4 weeks and their combination on the rat paw withdrawal pressure.

Results were represented as mean \pm SE (every group consisted of 6 rats)

$* p<0.05$ in comparison with control nondiabetic rats.

${ }^{\circledR} \mathrm{p}<0.05$ in comparison with STZ treated rats.

$\$ p<0.05$ in comparison with LIRA treated rats.

significant $(\mathrm{p}<0.05)$ increase in the rotarod staying time of diabetic rats in comparison with the streptozotocin treated group (Fig. 4). Treatment with vitamin $\mathrm{D}$ for 4 weeks causes an increase in the rotarod staying time of diabetic rats but this increase was nonsignificant in comparison with the streptozotocin treated group (Fig. 4). The combination of liraglutide and vitamin D caused a significant $(\mathrm{p}<$ 0.05) increase in the rotarod staying time of diabetic rats compared to the streptozotocin treated group (Fig. 4).

streptozotocin (STZ) $60 \mathrm{mg} / \mathrm{kg}$, liraglutide (LIRA) $0.8 \mathrm{mg} / \mathrm{kg} /$ day s.c.for 4 weeks, vitamin D (Vit D) $12 \mu \mathrm{g} / \mathrm{kg} / \mathrm{day}$ orally for 4 weeks and their combination on the rotarod staying time in rats.

Results were represented as mean \pm SE (every group consisted of 6 rats)

$* p<0.05$ in comparison with control nondiabetic rats.

${ }^{\circledR} \mathrm{p}<0.05$ in comparison with STZ treated rats. $\$_{\mathrm{p}}<0.05$ in comparison with LIRA treated rats.

3.2. Effect of single i.p. injection of streptozotocin (STZ) $60 \mathrm{mg} / \mathrm{kg}$, 
liraglutide (LIRA) $0.8 \mathrm{mg} / \mathrm{kg} / \mathrm{day}$ s.c.for 4 weeks, vitamin D (Vit D) 12 $\mu \mathrm{g} / \mathrm{kg} / \mathrm{day}$ orally for 4 weeks and their combination on the blood glucose concentration of rats.

The results demonstrated that there was a significant $(\mathrm{p}<0.05)$ increase in blood glucose in the rat group injected by streptozotocin60 $\mathrm{mg} / \mathrm{kg}$ (Fig. 5). Treatment with liraglutidefor 4 weeks caused a significant $(\mathrm{p}<0.05)$ reduction in the level of blood glucose in comparison with thestreptozotocin

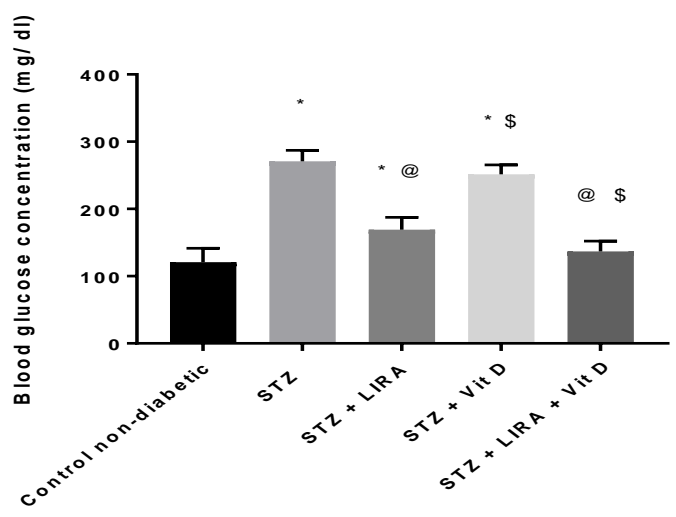

3.3. Effect of single i.p. injection of streptozotocin (STZ) $60 \mathrm{mg} / \mathrm{kg}$, liraglutide (LIRA) $0.8 \mathrm{mg} / \mathrm{kg} / \mathrm{day}$ s.c.for 4 weeks, vitamin D (Vit D) 12 $\mu \mathrm{g} / \mathrm{kg} / \mathrm{day}$ orally for 4 weeks and their combination on the serum level of tumor necrosis factor- $\alpha$ (TNF- $\alpha$ ) and serum level of interleukin-1 $\beta$ (IL-1ß) in rats.

Single-injection of streptozotocin 60 $\mathrm{mg} / \mathrm{kg}$ caused a significant $(\mathrm{p}<0.05)$ increase in the level of serum TNF- $\alpha$ in comparison with the control rat group (Fig.6A). Treatment with liraglutidefor 4 weeks caused a significant $(\mathrm{p}<0.05)$ reduction in the level of serum TNF- $\alpha$ in comparison with thestreptozotocin injected diabetic rats(Fig.6A). Oral administration of vitamin $D$ for 4 weeks caused a significant $(\mathrm{p}<0.05)$ reduction in the level of serum TNF- $\alpha$ in comparison with the streptozotocin treated group (Fig.6A). The combined use ofliraglutide and vitamin $\mathrm{D}$ for 4 weeks caused a significant $(p<0.05)$ injected rats(Fig.5). Oral administration of vitamin Dfor 4 weeks caused a slight reduction of the blood glucose of the diabetic rats and this reduction was non-significant in comparison with the streptozotocin treated group (Fig. 5). The combined use ofliraglutide and vitamin D3 for 4 weeks caused a significant $(\mathrm{p}<0.05)$ reduction in rat blood glucose and the reduction was significantly higher than that caused by the use of liraglutide alone (Fig. 5).

Fig.5 Effect of single i.p. injection of streptozotocin (STZ) $60 \mathrm{mg} / \mathrm{kg}$, liraglutide (LIRA) $0.8 \mathrm{mg} / \mathrm{kg} /$ day s.c. for 4 weeks, vitamin D (Vit D) $12 \mu \mathrm{g} / \mathrm{kg} /$ day orally for 4 weeks and their combination on the blood glucose concentration in rats.

Results were represented as mean \pm SE (every group consisted of 6 rats)

$* p<0.05$ in comparison with control nondiabetic rats.

${ }^{\circledR} \mathrm{p}<0.05$ in comparison with STZ treated rats. $\$ p<0.05$ in comparison with LIRA treated rats

reduction in the level of serum TNF- $\alpha$ compared to the streptozotocin treated group (Fig.6A). The reducing effect of this combination on TNF- $\alpha$ was much higher than the reduction caused byliraglutide alone and there was a statistical difference between the effects of the two groups on TNF- $\alpha$.

Regarding the effect on the serum level of IL-1 $\beta$, a single injection of streptozotocin $60 \mathrm{mg} / \mathrm{kg}$ caused a significant $(p<0.05)$ increase in the serumlevel of- $1 \beta$ compared to the control rat group (Fig.6B). Four weeks of treatment with liraglutideresulted in a significant $(p<0.05)$ reduction in the level of IL-1 $\beta$ of rat serum compared to thestreptozotocintreated rats(Fig.6B). Oral administration of vitamin D3 resulted in a significant $(\mathrm{p}<$ 0.05 ) reduction in the level of serum $\mathrm{IL}-1 \beta$ in comparison with the streptozotocin treated rats (Fig.6B). The combination ofliraglutide and vitamin D3, when used for 4 weeks, 
caused a significant $(\mathrm{p}<0.05)$ decrease in the level of IL-1 $\beta$ compared to the streptozotocin treated group (Fig.6B). Statistically, this combination caused a

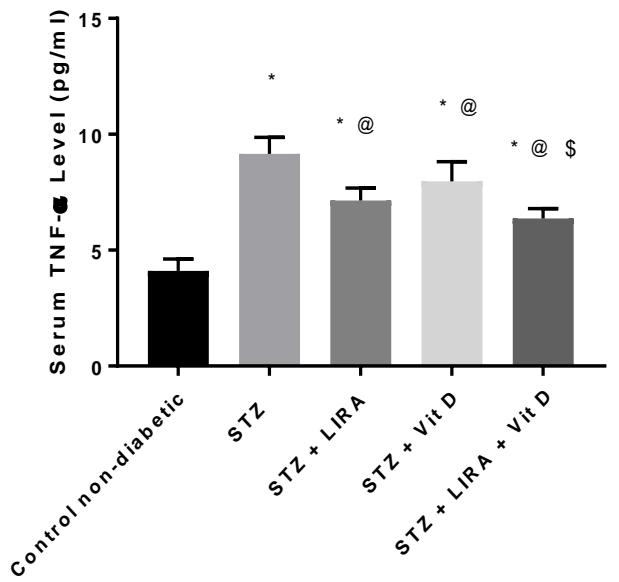

much reduction in the level of the elevated

IL-1 $\beta$ compared to the liraglutidetreated group.

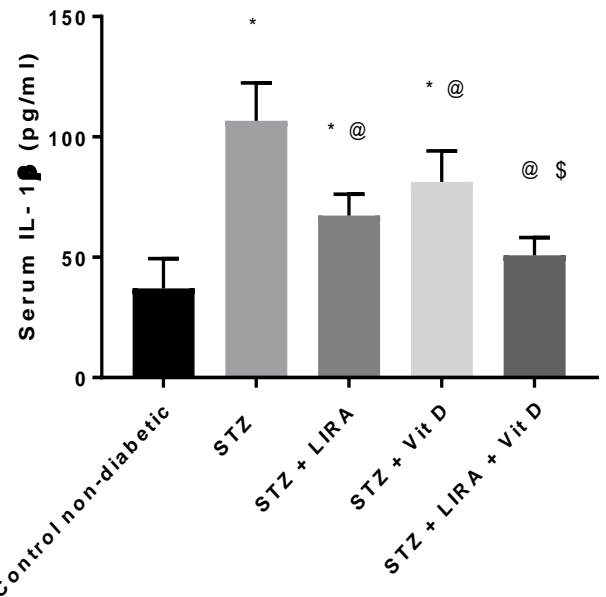

Fig.6 Effect of single i.p. injection of streptozotocin (STZ) $60 \mathrm{mg} / \mathrm{kg}$, liraglutide (LIRA) 0.8 $\mathrm{mg} / \mathrm{kg} /$ day s.c. for 4 weeks, vitamin D (Vit D) $12 \mu \mathrm{g} / \mathrm{kg} / \mathrm{day}$ orally for 4 weeks and their combination on the serum TNF- $\alpha(A)$ and serum IL-1 $(B)$ in rats.

Results were represented as mean \pm SE (every group consisted of 6 rats)

$* \mathrm{p}<0.05$ in comparison with control non-diabetic rats.

${ }^{\circledR} \mathrm{p}<0.05$ in comparison with STZ treated rats.

$\$ p<0.05$ in comparison with LIRA treated rats.

3.4. Effect of single i.p. injection of streptozotocin (STZ) $60 \mathrm{mg} / \mathrm{kg}$, liraglutide (LIRA) $0.8 \mathrm{mg} / \mathrm{kg} / \mathrm{day}$ s.c.for 4 weeks, vitamin D (Vit D) 12 $\mu \mathrm{g} / \mathrm{kg} / \mathrm{day}$ orally for 4 weeks and their combination on the level of malondialdehyde (MDA) and reduced glutathione (GSH) of rat sciatic nerve homogenate.

The results showed that a single i.p. injection of streptozotocin $60 \mathrm{mg} / \mathrm{kg}$ caused a significant $(\mathrm{p}<0.05)$ increase in the level of MDA of the rat sciatic nerve homogenate in comparison with the control rat group (Fig.7A). Treatment with liraglutide $0.8 \mathrm{mg} / \mathrm{kg}$ /day s.c.for 4 weeks caused a significant $(p<0.05)$ reduction in the level of the MDA compared to thestreptozotocin injected diabetic rats(Fig.7A). Treatment of the rats with oral vitamin D12 $\mu \mathrm{g} / \mathrm{kg} /$ day for 4 weeks caused a reduction in the level of MDA of sciatic nerve homogenate but this reduction was statistically nonsignificant in comparison with the streptozotocin treated group (Fig.7A). The use of the combination ofliraglutide and vitamin $\mathrm{D}$ for 4 weeks caused a significant $(\mathrm{p}<0.05)$ reduction in the level of MDA compared to the streptozotocin treated group (Fig.7A). The reducing effect of this combination on MDA was statistically much higher than the reduction caused byliraglutide alone.

Regarding the effect of the tested drugs on the level of GSH of rat sciatic nerve homogenate, the results showed that a single injection of streptozotocin 60 $\mathrm{mg} / \mathrm{kg}$ caused a significant $(\mathrm{p}<0.05)$ reduction in the level of GSH in comparison with the control rat group (Fig.7B). The use of liraglutide 0.8 $\mathrm{mg} / \mathrm{kg}$ /day for 4 weeks caused a significant $(p<0.05)$ elevation in the 
level of the GSH in comparison with thestreptozotocintreated rats(Fig.7B) and there was no significant difference in the level of GSH betweenliraglutide treated group and the control nondiabetic group. Oral vitamin D 12 $\mu \mathrm{g} / \mathrm{kg} /$ day for 4 weeks caused a nonsignificant increase in the level of GSH in comparison with the streptozotocin treated group (Fig.7B). The use of

A

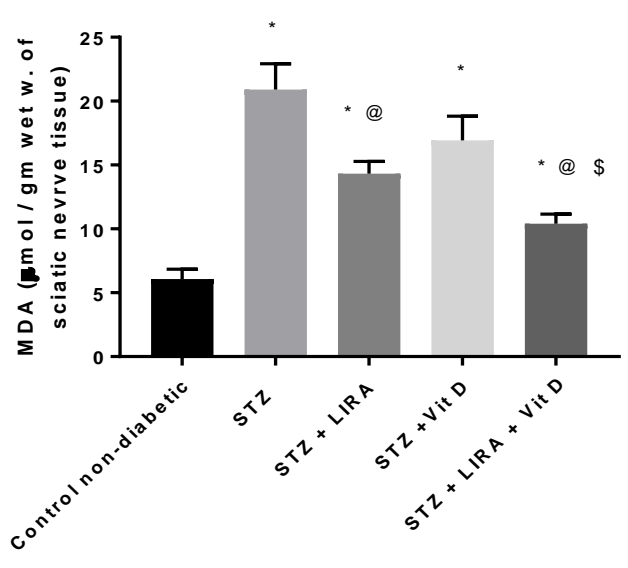

liraglutide in combination with vitamin D for 4 weeks caused a significant $(\mathrm{p}<$ $0.05)$ elevation in the level of GSH compared to the streptozotocin treated group (Fig.7B). There was no significant difference in the level of GSH between the control non-diabetic group and group treated by the combination of liraglutide with vitamin D.

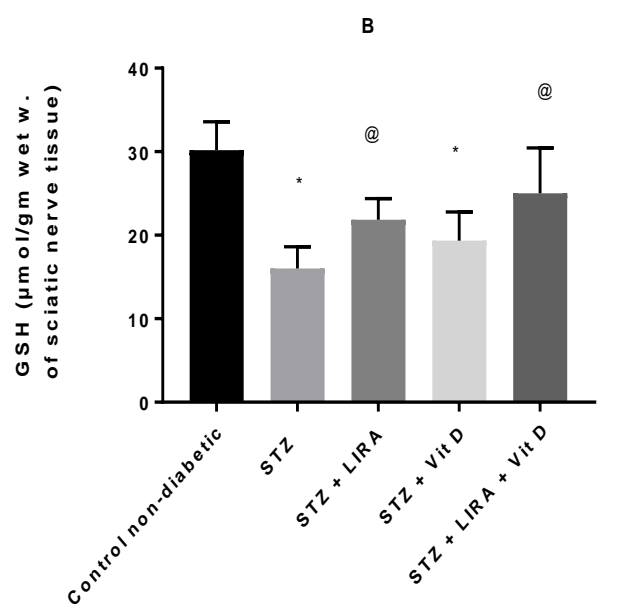

Fig.7 Effect of single i.p. injection of streptozotocin (STZ) $60 \mathrm{mg} / \mathrm{kg}$, liraglutide (LIRA) 0.8 $\mathrm{mg} / \mathrm{kg} / \mathrm{day}$ s.c. for 4 weeks, vitamin D (VitD) $12 \mu \mathrm{g} / \mathrm{kg} / \mathrm{day}$ orally for 4 weeks and their combination on MDA (A) and GSH (B) of rat sciatic nerve homogenate.

Results were represented as mean \pm SE (every group consisted of 6 rats)

$* \mathrm{p}<0.05$ in comparison with control non-diabetic rats.

${ }^{\circledR} \mathrm{p}<0.05$ in comparison with STZ treated rats.

$\$ p<0.05$ in comparison with LIRA treated rats.

\section{DISCUSSION}

More than $50 \%$ of patients with diabetes mellitus can develop diabetic neuropathy. Symptoms of neuropathy include; hyperalgesia, allodynia, and spontaneous pain [29]. Hyperalgesia is one of the characteristic symptoms of diabetic neuropathy in which there is a decrease in the reaction time to the thermal stimuli in diabetic rats. Painful neuropathy could be due to an increase inthe nociception and sensitivity caused by hyperglycemia [30]. Regarding the effect of the tested drugs on the rat behavioral tests, the results demonstrated that rats treated with streptozotocin $60 \mathrm{mg} / \mathrm{kg}$ developed thermal hyperalgesia as evident from the significant reduction of the hot plate and tail-flick latency after 4 weeks of diabetes induction. These results were in agreement with other reports that demonstrated that diabetic rats developed thermal and mechanical hyperalgesia after 4 weeks of streptozotocin injection [31, 32].

The results indicated that daily s.c. injection of liraglutideat a dose of 0.8 $\mathrm{mg} / \mathrm{kg}$ for 4 weeks caused a significant increase in the hot plate latency, tailflick latency, paw withdrawal pressure, and rotarod staying time of diabetic rats. These results were following other reports that demonstrated that treatment with liraglutide normalized the tail flick latency withdrawal time and the rotarod staying time of the 
diabetic rats [19].Liraglutide may exert its neuroprotection action through GLP-1 receptor that mediated activation of the adenylyl cyclase to increase cAMP production, which in turn stimulates protein kinase A (PKA) causingactivation and phosphorylation ofcAMP responseelement-binding protein (CREB) [33].

Treatment of the diabetic rats with 12 $\mu \mathrm{g} / \mathrm{kg} /$ day of vitamin D orally for 4 weeks caused a significant increase in the hot plate and tail-flick latency and a non-significant increase in the paw withdrawal pressure and rotarod staying time. These results demonstrated that vitamin $\mathrm{D}$ has a neuroprotective effect in the case of diabetic neuropathy which was following a study in humans that reported that oral administration of vitamin $D$ caused a significant reduction in the neuropathy symptom score in type 2 diabetic patients[34].Regarding the neuroprotective mechanism of vitamin D, several studies demonstrated that vitamin D can act on the neuronal cells through stimulation of the generation of several neurotrophins as nerve growth factor, glial cell line-derived neurotrophic factor andneurotrophin3 [35]. Modulation of neuronal calcium homeostasis and induction of the synthesis of calcium-binding protein, such as parvalbumin may contribute to the vitamin $\mathrm{D}$ neuroprotective effect [36].The results indicated that the combination of liraglutide in a dose of $0.8 \mathrm{mg} / \mathrm{kg}$ and vitamin D for 4 weeks caused a significant increase in the hot plate latency, tail-flick latency, paw withdrawal pressure, and rotarod staying time of diabetic rats. The effect was higher than the effect of liraglutide alone indicating that vitamin D3 can enhance the neuroprotective effect of liraglutide.

Regarding the effect of the tested drugs on the blood glucose, the results indicated that treatment of the rats with single i.p. injection of streptozotocin $60 \mathrm{mg} / \mathrm{kg}$ caused a significant increase in the blood glucose in comparison with the control group. The results were in agreement with other reports which demonstrated that a single injection of $60 \mathrm{mg} / \mathrm{kg}$ of streptozotocini.p.

causedhyperglycemia due to the induction of type-2 diabetes mellitus in rats [17]. Streptozotocin may act as a toxin for the pancreatic $\beta$-cell causing rapid destruction of the cells. Hyperglycemia induced by the use of streptozotocin can cause chronic endogenous oxidative stress [37]. Although the I.V. route or a large dose of streptozotocin caused a higher induction rate of diabetes compared to the i.p. route or the small dose, it may cause severe diabetes mellitus that impairs the general health of the rats. Gong et al. (2011) indicated that a dose of streptozotocin $60 \mathrm{mg} / \mathrm{kg}$ i.p. was the best choice for the induction of diabetic rat model without affecting the general health of the animal [17]. Injection of liraglutideat a dose of 0.8 $\mathrm{mg} / \mathrm{kg} /$ day for 4 weeks caused a significant reduction in the blood glucose level in comparison with the control diabetic group. These results were following the results of Yamazaki, et al. (2014) who indicated that the glucose level was significantly lower in diabetic rats treated with liraglutide than in the control ratgroup [38]. Regarding the mechanism of the anti-hyperglycemic effect of liraglutide, it causes stimulation of the secretion of insulin with a reduction of the secretion of glucagon [39]. The drug acts by binding to beta cells of the pancreas causing activation of adenylate cyclase leading to an increase in the intracellular cAMP. Finally, there is an activation of protein kinase- Aleading to stimulation of insulin secretion through exocytosis of 
the granules containing insulin[40].Liraglutide prevents also hypoadiponectinemia-induced increase in serum insulin, and serum lipids and decreases the impairment of hepatic and peripheral sensitivity to insulin. These liraglutide effects may be mediated through alterations in certain genes as Acrp30 and the expression of proteins involved in the metabolism of lipids and glucose [41].

Treatment of the diabetic rats with 12 $\mu \mathrm{g} / \mathrm{kg} /$ day of vitamin Dorally for 4 weeks caused a slight reduction of the blood glucose of the diabetic rats and this reduction was non-significant in comparison with the streptozotocin treated group. A similar study in diabetic rats reported that treatment with vitamin $\mathrm{D}$ either alone or in combination with insulin caused a significant reduction in serum glucose and insulin [42]. Another study reported that there was a reduction in the blood glucose level in rats supplemented with vitamin D [43]. The possible mechanism through which vitamin D can affect glucose levels could be caused by slow genomic effects of rapid non-genomic effects that stimulate the release of insulin by increasing the expression of vitamin D receptors [44]. The pancreatic gene expression of vitamin D receptors was enhanced by vitamin $D$ treatment in rats. It was found that the binding of vitamin D-receptor complex to the vitamin $\mathrm{D}$ response element in the insulin receptors leading to enhancement of the responsiveness of insulin for glucose transports [45].The combined use ofliraglutide and vitamin D for 4 weeks caused a significant reduction in rat blood glucose and the reduction was significantly higher than that caused by the use of liraglutide alone indicating that vitamin $\mathrm{D}$ can enhance blood glucose lowing effect of liraglutide.

Regarding the effect of the tested drugs on the proinflammatory markers, the results demonstrated that treatment of the rats with streptozotocin caused a significant increase in the serum level of TNF- $\alpha$ and IL- $1 \beta$ in comparison with the control group. The results were in agreement with other studies which indicated that there was an elevation in the proinflammatory markers such as IL-1 $\beta$, IL-6, and TNF$\alpha$ in the serum of streptozotocininduced diabetic rats in comparison with the control rats [46]. Hyperglycemia is one of the important factors that stimulate the generation of inflammatory markers in cases of diabetes mellitus through stimulation of the pathway of oxidative stress that leads to damage to nervous tissues [47]. The present findings demonstrated that there was an association between the inflammatory process and the development of diabetic neuropathy as there was an elevation in the serum level of TNF- $\alpha$ and Il-1 $\beta$ in diabetic rats. It is well documented that such cytokines have an important role in the pathogenesis of inflammation and the progression of the acute phase reaction [48]. Some reports indicated that the elevation in the level of inflammatory markers in diabetics is the result of insulin resistance and hyperglycemia [49].

In the present study, treatment of diabetic rats by liraglutide attenuated the elevated level of serum TNF- $\alpha$ and IL- $1 \beta$ which was in agreement with the results ofMoustafa et al. (2018) who demonstrated that liraglutide treatment caused a normalization of the inflammatory markers in the sciatic nerve homogenate of rats with diabetic neuropathy[19].Liraglutidecaused also a significant reduction of several proinflammatory markers, such as TNF- $\alpha$, IL-6, and plasminogen activator inhibitor type- 1 as well as reducing the serum levels of total cholesterol in rats[50]. 
The study indicated that the administration of vitamin $\mathrm{D}$ for 4 weeks caused a significant reduction in the level of TNF- $\alpha$ and IL- $1 \beta$ in comparison with the diabetic group. These changes were in agreement with the results ofEl-Sayed et al. (2015) who indicated that treatment with vitamin $D$ caused a significant decrease in serum IL-1ßof the diabetic rats with a significant increase in IL-10 which is an important antiinflammatory cytokine[42]. Vitamin D may affect the metabolism of glucose by decreasing the secretion of proinflammatory cytokines leading to a decrease in insulin resistance and a low level of vitamin D is associated with an increase in the level of C-reactive protein [51]. Chen et al. (2014) demonstrated that vitamin $\mathrm{D}$ retarded the development of diabetic neuropathy by decreasing the formation of pro-inflammatory markers such as TNF- $\alpha$, IL-6, and Creactive protein [52]. Vitamin D receptor signaling in the podocytes caused protection against diabetic neuropathy by affecting apoptotic pathways including the expression of caspase-3 and bcl-2[53]. The combined treatment by both liraglutide and vitamin D3 caused a significant reduction of the proinflammatory markers more than either treatment alone. This is an indicator of the presence of a synergistic effect between vitamin $\mathrm{D}$ and liraglutide therapy in decreasing the generation ofproinflammatory cytokines in diabetic rats.

Regarding the effect of the tested drugs on the sciatic nerve oxidative stress markers, the results demonstrated that treatment of the rats with streptozotocin caused a significant increase in the level of MDA with a significant reduction of the GSH in comparison with the control group. These results were in agreement with the results of Jorige and Akula (2015) who demonstrated that there was a significant reduction in the levels of GSH, catalase, and superoxide dismutase in the sciatic nerve of streptozotocin-induced diabetic neuropathy in rats. There was also a significant increase in the level of MDA which is the major lipid peroxidation end product [54].Hyperglycemia causes an increase in the oxidative stress in the peripheral nerves leading to the development of neuropathy. Glucose auto-oxidation, reduction in the antioxidant agents such as GSH and vitamin E, and impairment of the activity of antioxidant enzymes such as catalase, and superoxide dismutase are possible causes of oxidative stress in diabetic cases [55]. Chronic diabetes is characterized by lipid peroxidation. There is an increase in the generation of free radicals that interact with the cell membrane fatty acids causing peroxidation of lipid with excess formation of free radicals [56]. Low level of peroxide radicals causes stimulation of insulin secretion, however, excessive production of peroxide causes marked peroxidation of lipids leading to cellular infiltration and damage to pancreatic cells in diabetes mellitus type-1 [57].

The use of liraglutide for 4 weeks caused a significant reduction in the level of MDA with a significant elevation of the GSH in the sciatic nerve homogenate of the diabetic rats. Similar results indicated that treatment with liraglutide normalized the elevated levels of MDA and nitric oxide and increased the level of superoxide dismutase in the sciatic nerves of streptozotocin-induced diabetic neuropathy in rats [19]. It was reported that liraglutide has an important role as a neuroprotective and antioxidant agent against brain injury caused by ischemia by decreasing the 
MDA level and increasing GSH and superoxide dismutaseactivity [58]. Salcedo et al.(2012) reported that the antioxidant activity of liraglutide is the main mechanismresponsible for the neuroprotective effect of the drug. The nervous system is very vulnerable to oxidative stress injury due to the restriction in cell regeneration and the high rate of metabolism[59].

Treatment of diabetic rats with oral vitamin $\mathrm{D}$ for 4 weeks caused a reduction in the level of MDA with the elevation of the GSH in the sciatic nerve homogenate but these changes were statistically non-significant. The results were in agreement with the study that indicated that the administration of vitamin $\mathrm{D}$ alone or with insulin caused a significant reduction of hepatic MDA in diabetic rats [60]. Also another study demonstrated that oral vitamin $\mathrm{D}$ when combined with calcium caused a significant decrease in the level of MDAin diabetic rats with significant elevation in the activity of antioxidant enzymes such as glutathione peroxidase and catalase [61]. Regarding the mechanism through which vitamin $D$ causes activation of antioxidant enzymes, it may be due to an increase in the formation of metal ions such as $\mathrm{Zn}$ and $\mathrm{Cu}$ which act as cofactors essential for the activity of antioxidant enzymes [62]. There was a significant reduction in the level of the markers of oxidative stress such as MDA and nitric oxide with a signifycant elevation in the level of antioxidant enzymes such as superoxide dismutase and catalase after treatment of patients in hemodialysis with paricalcitol which is a vitamin $D$ receptor activator [63]. Treatment with vitamin $D$ caused an increase in renal levels of the antioxidant enzymes such as superoxide dismutase and GSH leading to a marked decrease in renal reactive oxygen species generation that helps in the inhibition of renal ischemia [64]. The adding of vitamin $\mathrm{D}$ to endothelium before the induction of oxidative stress can enhance the survival of the cells [65]. Treatment of diabetic rats with the combination of liraglutide and vitamin $\mathrm{D}$ caused a significant reduction of the oxidative stress markers more than either treatment alone. This is an indicator of the presence of a synergistic effect between vitamin $\mathrm{D}$ and liraglutide therapy in the normalization of the markers of oxidative stress in the sciatic nerve homogenate of the diabetic rats.

\section{CONCLUSION}

The study had shown that liraglutidehas a moderate neuroprotective effect in cases of streptozotocin-induced diabetic neuropathy in rats with moderate anti-inflammatory and antioxidant effects that may be responsible for its neuroprotective effects. Vitamin D has mild anti-inflammatory and antioxidant effects which may be responsible for its mod-est neuroprotective effect. The addition of vitamin $\mathrm{D}$ to liraglutideshows enhanceement of the neuroprotective, antiinflammatory, and antioxidant effects of liraglutide.

\section{Funding}

This research did not receive any grant.

\section{Conflict of Interests}

The author declares no conflict of interest in preparing this research.

\section{Reference}

1.L. Vileikyte, R.R. Rubin, H.Leventhal, Psychological aspects of diabetic neuropathic foot complications: an overview. DiabetesMetab. Res. Rev. 20: S13-S18 (2004).

2. L. Chen, B.Li, B. Chen, Y. Shao, Q.Luo, X.Shi, et al., Thymoquinone alleviates the experimental diabetic peripheral neuropathy by modulation of inflammation. Sci. Rep. 6:1-11 (2016).

3. Y. Oh, Bioactive compounds and their neuroprotective effects in diabetic complications. Nutrients 8:1-20(2016). 
4. S.Soheilykhah, M.Rashidi, F.Dehghan, A.Shojaoddiny-Ardekani, S.RahimiSaghand, Prevalence of peripheral neuropathy in diabetic patients. Iran J. Diabetes Obes. 5: 107-113(2013).

5. J.L.Rains, S.K.Jain, Oxidative stress, insulin signaling, and diabetes.FreeRadic. Biol. Med. 50 (5): 567-575(2012).

6. D.Russell-Jones, Molecular, pharmacological, and clinical aspects of liraglutide, a once-daily human GLP-1 analog.Mol. Cell Endocrinol. 297:137-140 (2009).

7. H.Agerso, L.B.Jensen, B.Elbrond, P.Rolan, M.Zdravkovic, The pharmacokinetics, pharmacodynamics, safety, and tolerability of NN2211, a new long-acting GLP-1 derivative in healthy men.Diabetologia 45:195-202 (2002).

8. D.J.Drucker, M.Nauck, The incretin system: Glucagon-like peptide-1 receptor agonists and dipeptidyl peptidase-4 inhibitors in type 2 diabetes. Lancet 368:1696-1705(2006).

9. M.Horowitz, A.Flint, K.L.Jones, C.Hindsberger, M.F.Rasmussen, C.Kapitza, et al. , Effect of the oncedaily human GLP-1 analog liraglutide on appetite, energy intake, energy expenditure and gastric emptying in type 2 diabetes. Diabetes Res. Clin.Pract. 97(2), 258-266(2012).

10. A.M.Borissova, T.Tankova, G.Kirilov, L.Dakovska, R. Kovacheva, The effect of vitamin D3 on insulin secretion and peripheral insulin sensitivity in type 2 diabetic patients. Int. J.Clin.Pract. 57: 258-261(2003).

11. O.Gedik, S.Akalin, Effects of vitamin D deficiency and repletion on insulin and glucagon secretion in man.Diabetologia 29:142-145(1986).

12. N.G.Forouhi, J.Luan, A.Cooper, B.J.Boucher, N.J.Wareham, Baseline serum 25-hydroxy vitamin $\mathrm{D}$ is predictive of future glycemic status and insulin resistance: the Medical Research Council Ely Prospective Study 1990-2000.Diabetes 57: 26192625(2008).

13. B.S.Chertow, W.I.Sivitz, N.G.Baranetsky, M.B.Cordle, H.F. DeLuca, Islet insulin release, and net calcium retention in vitro in vitamin Ddeficient rats. Diabetes 35: 771775(1986).

14. L.G.Danescu, S.Levy, J.Levy, Vitamin $\mathrm{D}$, and diabetes mellitus. Endocrine 35:11-17(2009).

15. P.Shillo, D.Selvarajah, M. Greig, R .Gandhi, G .Rao, I.D Wilkinson, et al. , Reduced vitamin D levels in painful diabetic peripheral neuropathy. Diabet. Med. 36(1) ,44-51(2019).

16. A.Ghadiri-Anari, Z. Mozafari, S. Gholami, S.A. Khodaei, M.Aboutorabi-Zarchi, F .Sepehri, et al. , Dose vitamin D supplementations improve peripheral diabetic neuropathy? A before-after clinical trial.DiabetesMetab.Syndr. 13(1) ,890893(2019).

17. Y.H Gong, X.R.Yu, H.L.Liu, N Yang, P.P.Zuo, Y.G. Huang, Antinociceptive effects of the combination of tramadol and acetaminophen on painful diabetic neuropathy in streptozotocin-induced diabetic rats. Acta Anaesthesiol.Taiwan49(1),1620(2011).

18. W.T.Lu, J.H. Juang, B.R. Hsu, H.S.Huang, Effects of high or low dose of streptozocin on pancreatic islets in C57BL/6 and C.B17-SCID mice. Transplantation proceedings 30(2),609610(1998).

19. P.E. Moustafa, N.F. Abdelkader, S.A. El Awdan, O.A.El-Shabrawy, H.F.Zaki, Liraglutide ameliorated peripheral neuropathy in diabetic rats: Involvement of oxidative stress, inflammation, and extracellular matrix remodeling.JNeurochem. 146(2),173185(2018).

20. N.George, T.P Kumar, S.Antony, S.Jayanarayanan, C.S Paulose, Effect of vitamin D3 in reducing metabolic and oxidative stress in the liver of streptozotocin-induced diabetic rats.Br. J.Nutr. 108(8),1410-8(2012).

21. S.Hunskaar, O.G.Berge, K.Hole, A modified hot-plate test sensitive to mild analgesics.Behav. Brain Res. 21:101108 (1986.).

22. S. K.ugimoto, I.B.Rashid, M.Shoji, T.Suda, M.Yasujima, Early changes in insulin receptor signaling and pain 
sensation in streptozotocin-induced diabetic neuropathy in rats. J. Pain 9: 237-245(2008).

23. L.O.Randall, J.J Selitto, A method for measurement of analgesic activity on inflamed tissue. Arch.Int. Pharmacodyn.Ther. 111: 409419(1957).

24. S.M.Cartmell, L.Gregor, D.Mitchell, A revised rotarod procedure for measuring the effect of antinociceptive drugs on motor function in the rat. J. Pharmacol. Methods 26: 149159(1991).

25. D.Braham, , P.Tinder, An improved color reagent for the determination of blood glucose by the oxidase system. Analyst 97: 142-145(1972).

26. C.A.Ismail, C.B. AbdAziz, R. Suppian, I.Long, Imbalancedoxidativestressandproinflammatory markers differentiate the development of diabetic neuropathy variants in streptozotocin-induced diabetic rats.J.Diabetes Metab.Disord.17:129-136(2018).

27. H.Ohkawa, N.Ohishi, K.Yagi, Assay for lipid peroxides in animal tissues by thiobarbituric acid reaction. Anal.Biochem. 95: 351-358 (1972).

28. A.F.Boyne, G.L.Ellman, A methodology for the analysis of tissue sulfhydryl components.Anal.Biochem. 46(2),639-653(1972).

29. S.C.Apfel, A.K.Asbury, V.Bril, T.M Burns, J.N.Campbell, C.H.Chalk, et al., Positive neuropathic sensory symptoms as endpoints in diabetic neuropathy trials. J. Neurol. Sci. 189: 3-5(2001).

30. D. Fuchs, F.Birklein, P.W.Reeh, S.K.Sauer, Sensitized Peripheral Nociception in Experimental Diabetes of the Rat. Pain 151: 496-505(2010).

31. A.Fox, C. Eastwood, C.Gentry, D. Manning, L. Urban, Critical evaluation of the streptozotocin model of painful diabetic neuropathy in the rat.Pain 81(3), 307-316(1999).

32. M.Malcangio, D.R. Tomlinson, A pharmacologic analysis of mechanical hyperalgesia in streptozotocin/diabetic rats.Pain76(1-2), 151-157(1998).

33. Y.Bao, L.Jiang, H .Chen, J .Zou, Z.Liu, Y. Shi, The Neuroprotective
Effect of Liraglutide is Mediated by Glucagon-Like Peptide 1 ReceptorMediated Activation of cAMP/PKA/CREB Pathway. Cell Physiol.Biochem.36(6),2366-

2378(2015).

34. D.Shehab, K.Al-Jarallah, N.Abdella, O.A.Mojiminiyi, H .Al Mohamed, Prospective evaluation of the effect of short-term oral vitamin D supplementation on peripheral neuropathy in type 2 diabetes mellitus. Med.Princ. Pract.24:250-256(2015).

35.M.S.Saporito, E.R.Brown, K.C. Hartpence, H.M. Wilcox, J.L.Vaught, S.Carswell, Chronic 1,25dihydroxyvitamin D3-mediated induction of nerve growth factor mRNA and protein in L929 fibroblasts and in adult rat brain. Brain Res.633:189-196(1994).

36. P. A .de Viragh, K.G.Haglid, M.R.Celio, Parvalbumin increases in the caudate-putamen of rats with vitamin D hypervitaminosis. Proc. Natl. Acad. Sci. USA 86: 3887-3890(1989).

37. K.Hayashi, R.Kojima, M.Ito, Strain differences in the diabetogenic activity of streptozotocin in mice. Biol. Pharm. Bull. 29(6),1110-1119(2006).

38. S. Yamazaki, H. Satoh, T.Watanabe, Liraglutide enhances insulin sensitivity by activating AMP-activated protein kinase in male Wistar rats. Endocrinol. 155(9),3288-3301(2014).

39. L.M de MesquitaAraujo, S. Feguri, T. L .de Oliveira, F.B. Pedrosa, R.G Guimaraes, L.B.P.C de Sa., et al., Extra glycemic impacts of GLP-1 receptor agonists: benefits of a class effect? Open J. Endocr. Metab. Dis. 6:4357(2016).

40. P. E MacDonald, W.El-Kholy, M.J.Riedel, A.M.F.Salapatek, P.E. Light, M.B. Wheeler, The multiple actions of GLP-1 on the process of glucose-stimulated insulin secretion. Diabetes $51 \quad$ (Suppl 3): S434S442(2002).

41.L.Li, Z. Miao, R. Liu, M.Yang, H.Liu, G.Yang, Liraglutide prevents hypoadiponectinemia induced insulin resistance and alterations of gene expression involved in glucose and lipid metabolism. Mol. Med. 17(11- 
12), 1168-1178(2011).

42. L.A. El-Sayed, O.M.Tork, H. Seddiek, R.M.Taha, N.I.Gomaa, Vitamin D protects diabetic rats from neuropathic changes by improving insulin sensitivity and upregulating vitamin D receptors. Kasr Al Ainy Medical Journal 21:115-124(2015).

43. S.Gregori, N.Giarratana, S. Smiroldo, M. Uskokovic, L.A. Adorini, 1alpha,25dihydroxyvitamin D (3) analog enhances regulatory $\mathrm{T}$-cells and arrests autoimmune diabetes in NOD mice. Diabetes 51:1367-1374(2002).

44. D. Bikle, Nonclassic actions of vitamin D. J. Clin.Endocrinol. Metab.94:26-34(2009).

45. D.Zehnder, R.Bland, M.C.Williams, R.W. McNinch, A.J. Howie, P.M.Stewart, et al., Extrarenal expression of 25-hydroxyvitamin d(3)1 alpha-hydroxylase. J. Clin.Endocrinol. Metab.86:888894(2001).

46. M.M.Al-Enazi, Protective Effects of Combined Therapy of Rutin with Silymarin on Experimentally-Induced Diabetic Neuropathy in Rats.Pharmacology\& Pharmacy 05(09),876-889(2014).

47. J.Li, G.H.Wei, H.Huang, Y.P.Lan, B.Liu, H.Liu, et al., Nerve InjuryRelated Autoimmunity Activation Leads to Chronic Inflammation and Chronic Neuropathic Pain. Anesthesiology, 118: 416-429(2013).

48. R.M.Locksley, N.Killeen, M.J.Lenardo, The TNF and TNF Receptor Superfamilies: Integrating Mammalian Biology. Cell 104: 487501(2001).

49. M., Brownlee, The Pathobiology of Diabetic Complications: A Unifying Mechanism. Diabetes 54: 16151625(2005).

50. N. Guo, J.Sun, H.Chen, H.Zhang, Z. Zhang, D. Cai, , Liraglutide prevents diabetes progression in prediabetic OLETF rats. Endocrine J. 60(1), 1528(2013).

51. M.Amer, R.Qayyum, Relation between serum 25-hydroxyvitamin D and Creactive protein in asymptomatic adults (from the continuous National Health and Nutrition Examination Survey
2001 to 2006). Am. J. Cardiol.109:226230(2012).

52. N.Chen, Z.Wan, S.F.Han, B.Y.Li, Z.L.Zhang, L.Q.Qin, Effect of Vitamin D Supplementation on the Level of Circulating High-Sensitivity CReactive Protein: A Meta-Analysis of Randomized Controlled Trials. Nutrients 6: 2206-2216(2014).

53. Y.Wang, D.K. Deb, Z. Zhang, T., Sun, W.Liu, D.Yoon, et al., Vitamin D Receptor Signaling in Podocytes Protects against Diabetic Nephropathy. J. Am. Soc. Nephrol.23(12), 19771986(1986).

54. A.Jorge, A.Akula, Neuroprotective Role of Wheatgrass Powder in Experimental Diabetic Neuropathy via Modulating Oxidative Stress Markers in Rat Sciatic Nerves. Am. J. Phytomed. Clin.Ther.3(7),529540(2015)

55. A.M.Vincent, J.W.Russell, P.Low, E.L Feldman, Oxidative stress in the pathogenesis of diabetic neuropathy.Endocr. Rev. 25(4),612628 (2004).

56. U. Levy, H.Zaltzber, A.Ben-Amotz, Y. Kanter, M. Aviram, Carotene affects antioxidant status in non-insulin dependent diabetes mellitus. Pathophysiol.6:157-161(1999).

57. S.A.Metz, Oxygenation products of arachidonic acid: third messengers for insulin release. Prostaglandins 27:147151(1984).

58. S.Briyal, S. Shah, A.Gulati, Neuroprotective, and antiapoptotic effects of liraglutide in the rat brain following focal cerebral ischemia. Neuroscience 281: 269-281(2014).

59. I.Salcedo, D.Tweedie, Y.Li, N.H.Greig, Neuroprotective and neurotrophic actions of glucagon-like peptide-1: an emerging opportunity to treat neurodegenerative and cerebrovascular disorders. Br. J. Pharmacol. 166: 1586-1599(2012).

60. N.George, T.P. Kumar, S.Antony, S.Jayanarayanan, C.S. Paulose,

Effect of vitamin D3 in reducing metabolic and oxidative stress in the liver of streptozotocin-induced diabetic rats.Br. J.Nutr. 108(8), 14101418(2012). 
61. F.S.Alatawi, U.A.Faridi, M.S.Alatawi, Effect of treatment with vitamin D plus calcium on oxidative stress in streptozotocin-induced diabetic rats SaudiPharm.J.26:1208-1213(2018).

62. T.Claro da Silva, C. Hiller, Z. Gai, G.A.Kullak-Ublick, Vitamin D3 transactivates the zinc and manganese transporter SLC30A10 via the vitamin D receptor. J. Steroid Biochem. Mol. Biol. 163: 77-87(2016).

63. M.J. Izquierdo, M.Cavia, P.Muniz, A.L.de Francisco, M.Arias, J. Santos, et al., Paricalcitol reduces oxidative stress and inflammation in hemodialysis patients. BMC
Nephrol.13:159(2012).

64. G.Sezgin, G.Öztürk, Ş.Güney, O.Sinanoğlu, M.Tunçdemir, Protective effect of melatonin, and 1, 25dihydroxyvitamin D3 on renal ischemia-reperfusion injury in rats.Ren. Fail. 35:374-379(2013).

65. F. Uberti, D.Lattuada, V.Morsanuto, U.Nava, G.Bolis, G.Vacca, et al., Vitamin D protects human endothelial cells from oxidative stress through the autophagic and survival pathways. J.Clin.Endocrinol.Metab. 99:13671374(2014). 